\title{
Research Paper: Effect of Cognitive Task on Gait Balance in People With Functional Ankle Instability
}

\section{Saeed Forghany ${ }^{1}$, Sanam Tavakoli Oskoei}

1. Musculoskeletal Research Centre, Isfahan University of Medical Sciences, Isfahan, Iran.

2. Department of Physical Therapy, School of Rehabilitation Sciences, Isfahan University of Medical Sciences, Isfahan, Iran.

$\begin{gathered}\text { Use yourdevice toscan } \\ \text { and read the article online }\end{gathered}$
ity (Persian)]. Archives of Rehabilitation. 2017; 18(3):220-229. http://dx.doi.org/10.21859/jrehab-1803220
dol: : http://dx.doi.org/10.21859/jrehab-1803220

Received: 7 Apr. 2017

Accepted: 3 Aug. 2017

Keywords:

Ankle sprain, Atten-

tion, Cognition, Dy-

namic balance, Gait

\section{ABSTRACT}

Objective Some individuals with Chronic Ankle Instability (CAI) termed as functional ankle instability (FAI) suffer from repetitive ankle giving way and feeling of ankle joint instability during dynamic activities like walking. Walking, as a postural task, requires some central attention to integrate sensory inputs, estimate, and plan and produce proper motor outputs. Attention demanding cognitive task has the ability to influence walking control and may increase the risk of giving way and ankle sprain. The purpose of this study was to investigate the effect of dual-tasking on dynamic balance in people with FAl.

Materials \& Methods Twelve physically active with clinically diagnosed FAl and 12 matched controls completed trials of normal walking in isolation or with a concurrent cognitive task, which is repeatedly subtracting seven from a randomly selected number (between 200 and 250) and the same cognitive task while sitting. Spatiotemporal parameters (measured by a seven-camera motion capture system) were calculated by visual3D during gait cycles. Gait velocity, step time, step length, stance time, and swing time were calculated. Independent t-test was used to compare the data for FAI and control groups, and comparisons between the single and dual task conditions were made using the paired t-test. Step time variability was calculated using Intraclass Correlation (ICC).

Results The results indicated that step velocity was decreased and that stance, swing, and step time were increased significantly during dual task walking in FAI people $(P<0.05)$. FAl people demonstrated greater step time variability during single and dual tasks compared to the control group.

Conclusion The athletes with FAl demonstrated different movement strategies during the dual-task condition compared to control group. Cognitive load may increase the risk of ankle instability in individuals with FAI.

\section{* Corresponding Author:}

Sanam Tavakoli Oskoei, MSc.

Address: Department of Physical Therapy, School of Rehabilitation Sciences, Isfahan University of Medical Sciences, Isfahan, Iran.

Tel: +98 (914) 3065660

E-Mail: tavakoli.sanampt@gmail.com 


\title{
اثر فعاليت شناختى بر تعادل راهرفتن در افراد مبتلابه بىثباتى عملكردى مج يا
}

\author{
سعيد فرقانى'، "صنم توكلى اسكويى' \\ ا - إكز تحقيقات اسكلتى عضلاتى، دانشكاه علوم يزٔشكى و خدمات بهداشتى درمانى اصفهان، اصفهان، ايران.

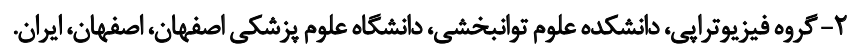

\begin{abstract}
حكSe

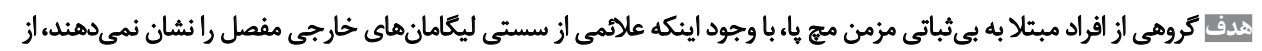

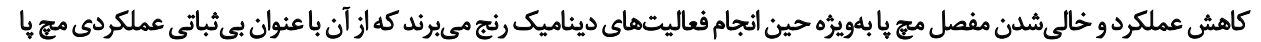

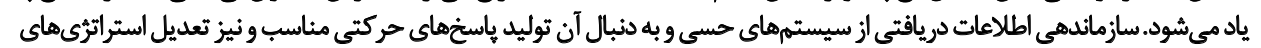

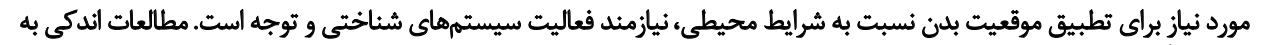

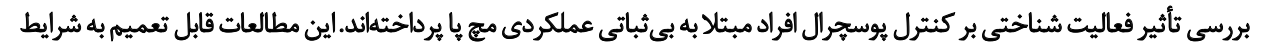

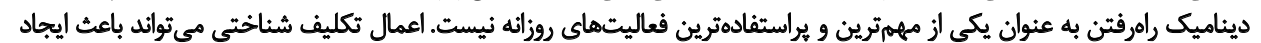

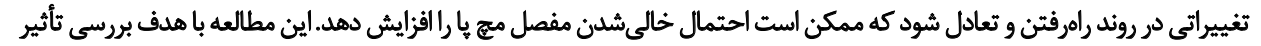

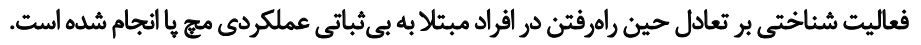

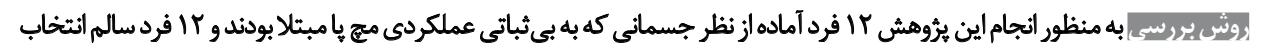

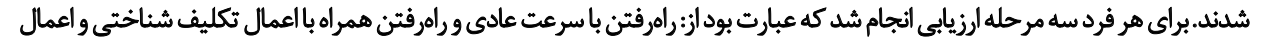

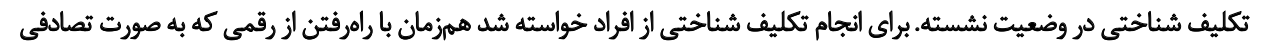

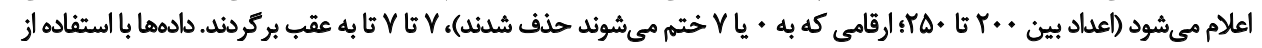

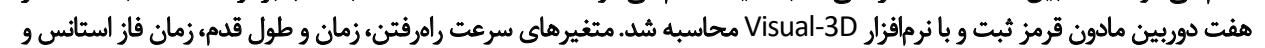

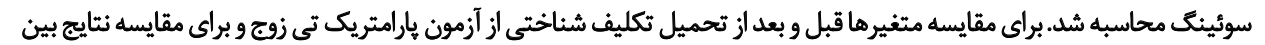

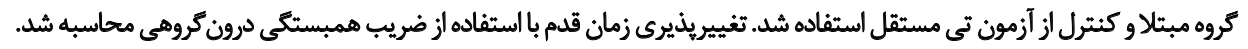

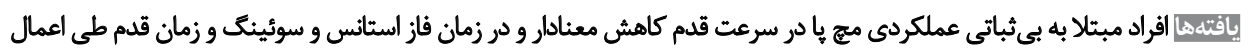

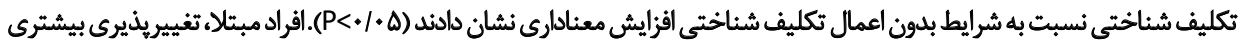

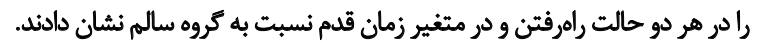

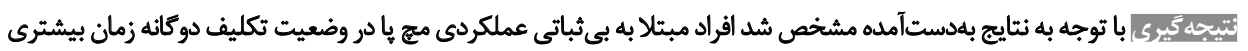

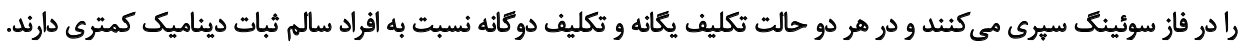

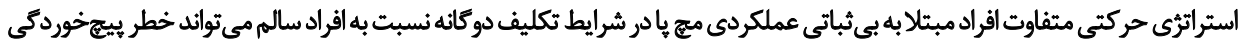

واريخ دريافت: A 14 فروردين

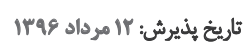

مزمن مج ياّ مطرح شده است. آسيب عوامل مكانيكى مفصل

daleo

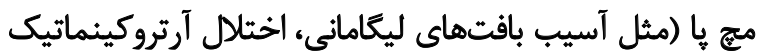

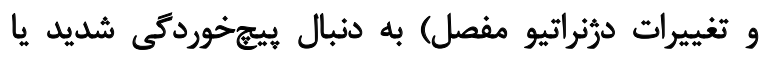

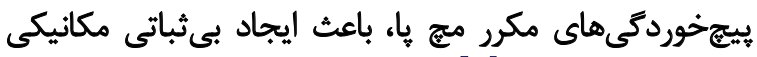

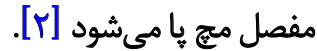

كروهى از افراد مبتلا به بـثباتى مزمن مج ها، با وجود اينكه

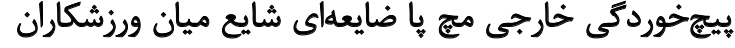

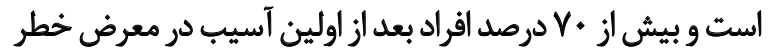

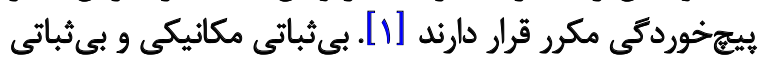

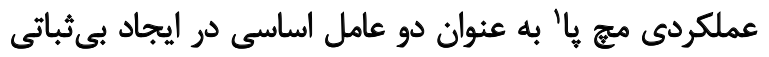


انجام شود و نيازمند بيش از كل ظرفيت باشد، تداخل ايجاد

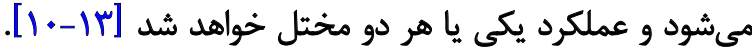

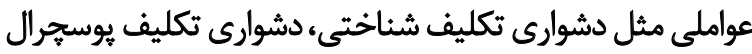

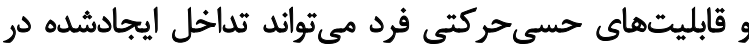

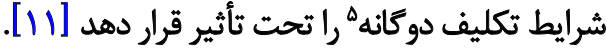

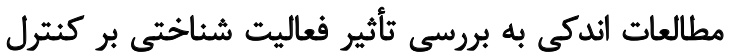

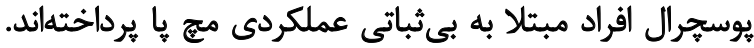

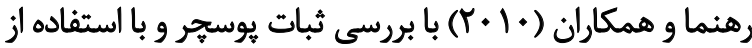

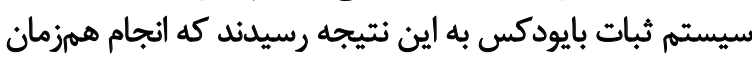

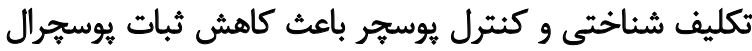

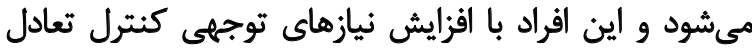

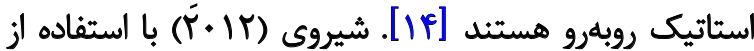
صفحه نيرو كزارش كرد كه اعمال تكليف شناختى حين كنتري

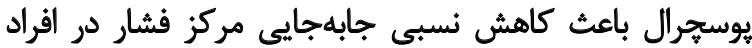

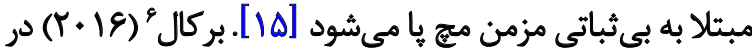

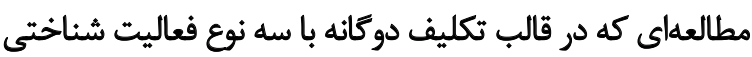

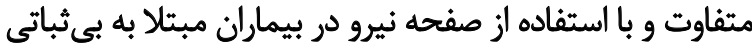

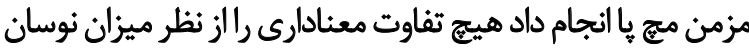

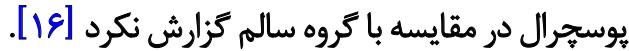

با وجود تفاوت در نتايج كزارششده، اين مطالعات قابل

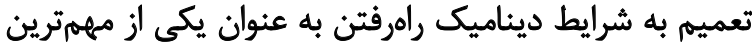

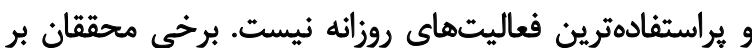

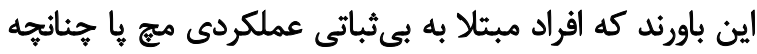

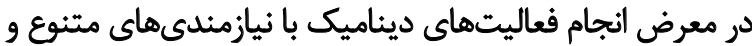

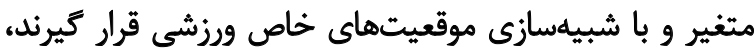

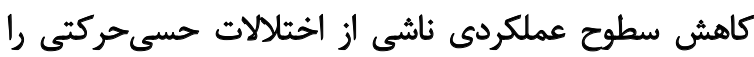

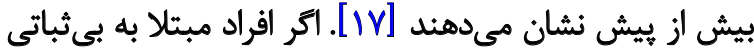

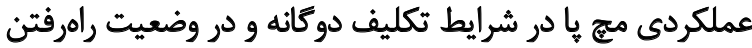

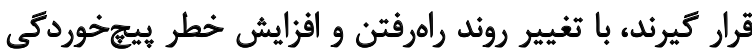

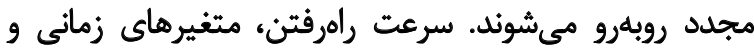

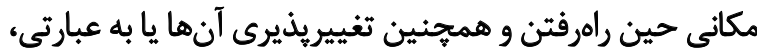

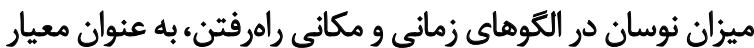

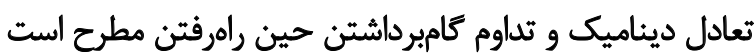

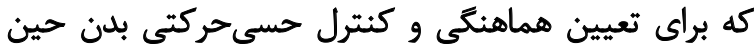

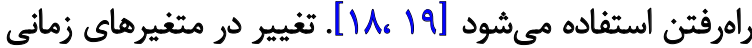

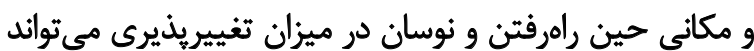

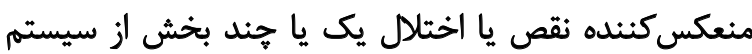

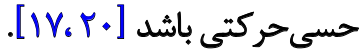

يككى از مههمترين اهداف توانبخشى افراد مبتلا به بي بيثباتى

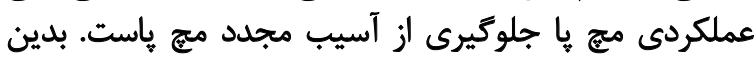

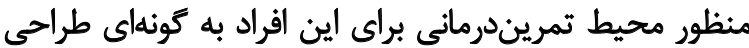

5. Dual task

6. Burcal
علائمى از سستى ليكامان هاى خارجي مفصل را نشان نمي دهند،

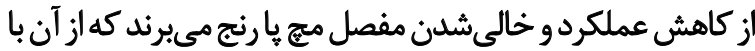

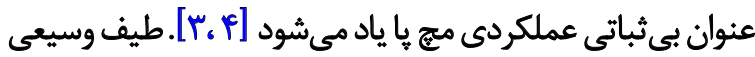

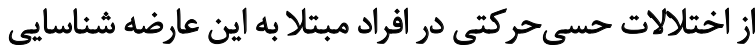

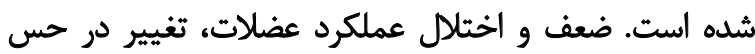
حركت و نيرو و حس موقعيت مفصل، تغيير در سازوكار كنترل فئرل

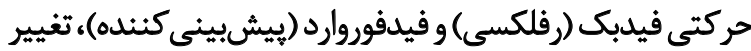

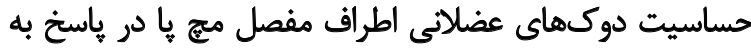

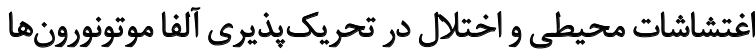

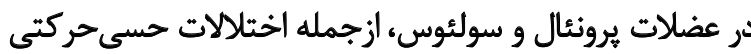

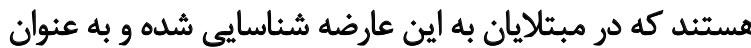

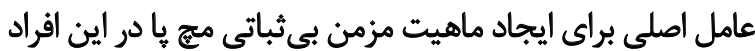

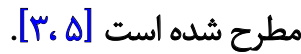

بيجز خوردگى مج يا در شرايط ديناميك وطى انجام فعاليتهاى

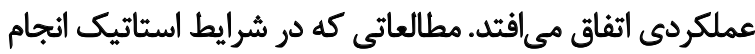

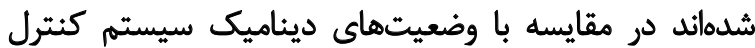

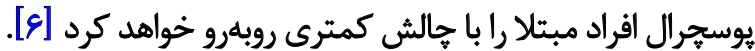

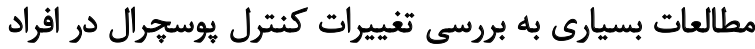

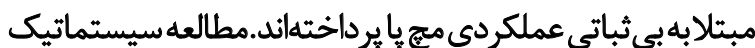

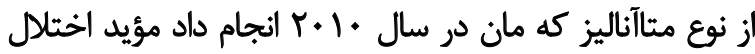

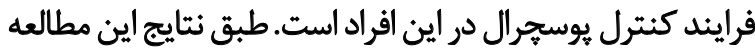

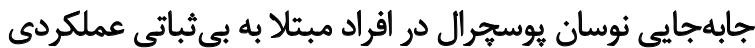

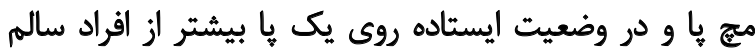

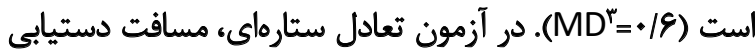

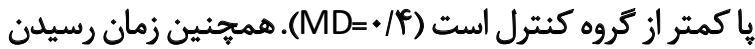

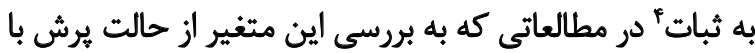

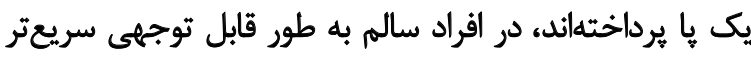

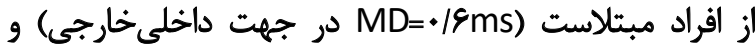

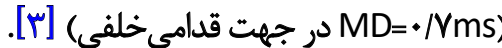

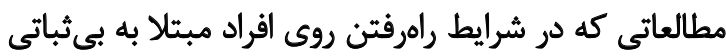

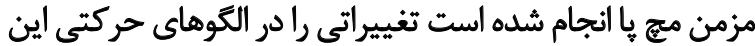

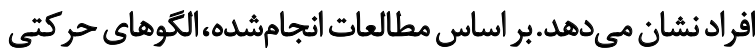

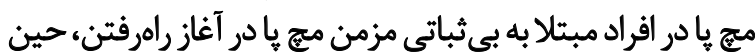

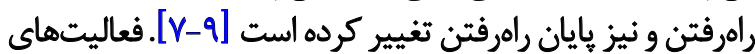

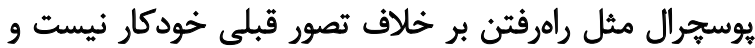

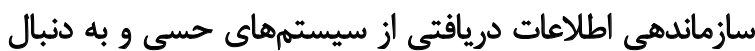

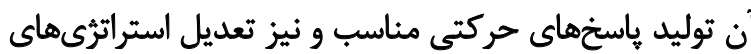

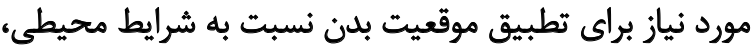

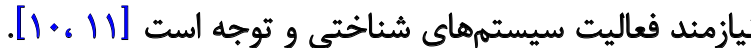

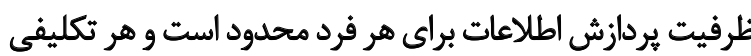

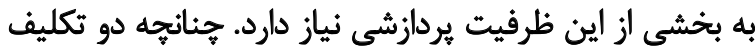
حسى حركتى (مثل راهرفتن) و تكليف شناختى به صورت همز نازمان

3. Standardized Mean Differences (MD)

4. Time to stabilization 
به مطالعه شامل بروز حداقل Y بار خالىشدن و يا بيجخ بحردمتى

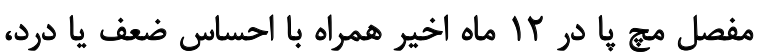

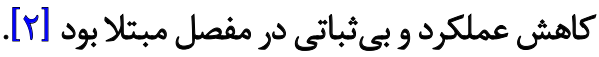

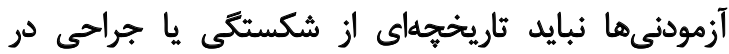

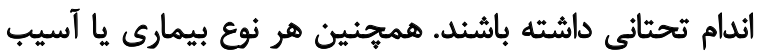

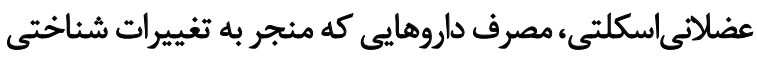
يا تعادلى شود، علائم حاد در زمان مطالعه، آسيب اندام تحان تحتاني

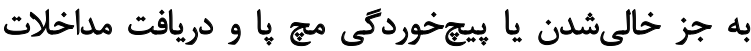

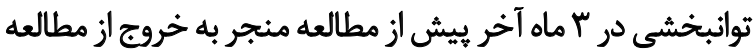

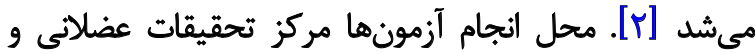

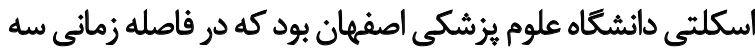

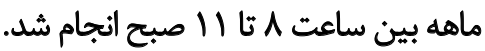
براى ثبت اطلاعات زمانى و مكانى راهرفتن از هفت دوربين

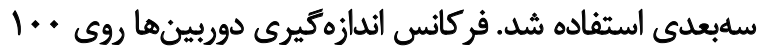

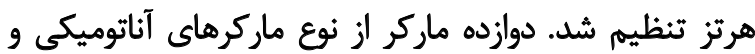

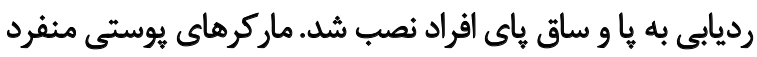

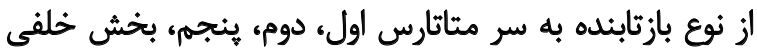

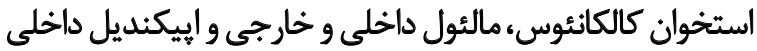

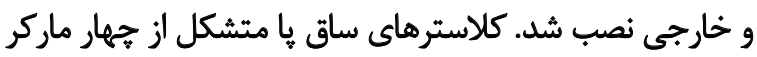
در أل If

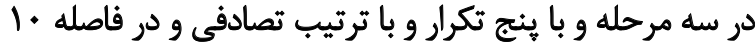

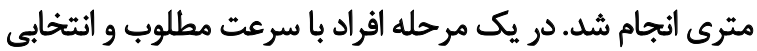

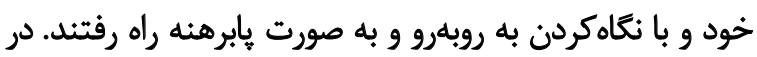

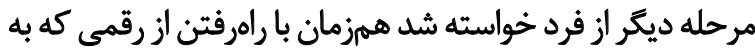

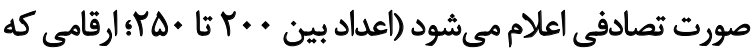

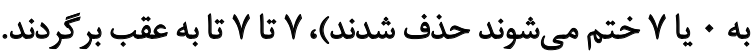

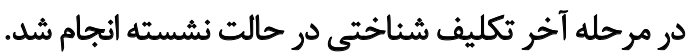

Visual-3D بس از انتقال دادههاى خام كينماتيكي به نرمافزار

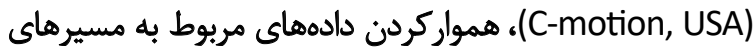

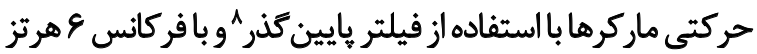

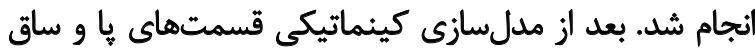

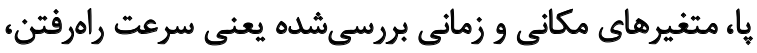

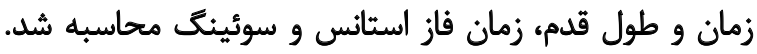
تجزيهوتحليل يافتهها به كمك نرمافزئ فار

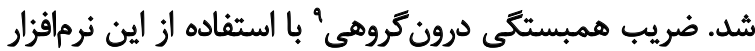

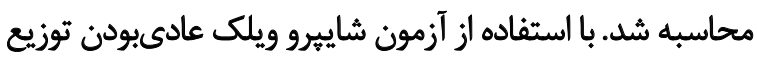

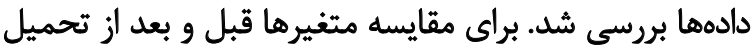

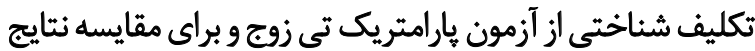

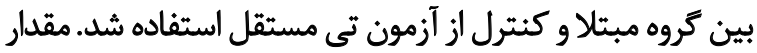

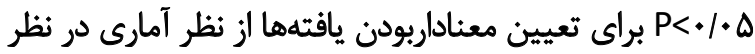

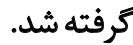

8. $4^{\text {th }}$ Order butterworth low-pass 9. Intraclass Correlation Coefficient (ICC)
مىشود كه با استفاده از فيدبكهاي متغير حسى و اعمال تكاليف

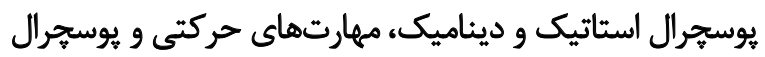

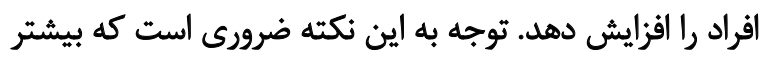

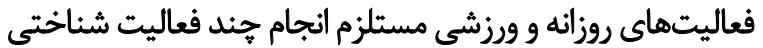

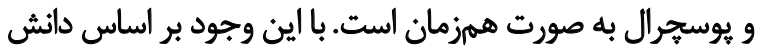

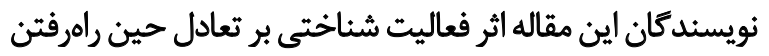

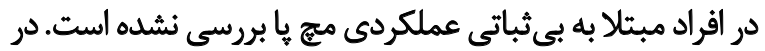

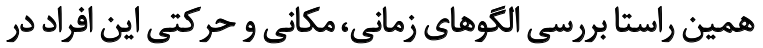

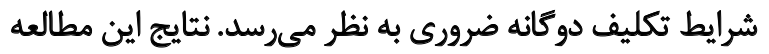

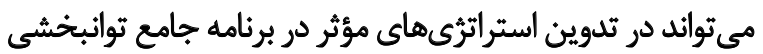

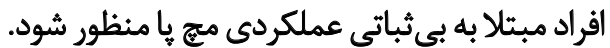

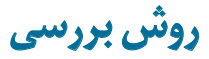

در اين مطالعه ابتدا هاع فرد از ميان مراكز ورزشى استان

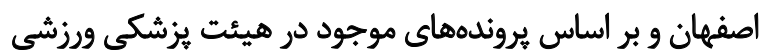

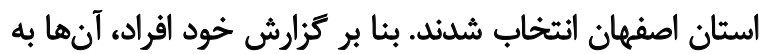

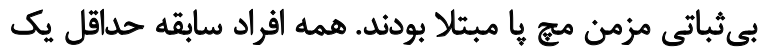

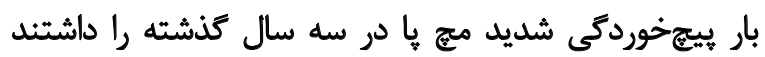

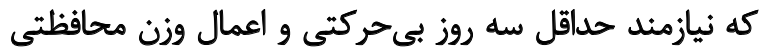

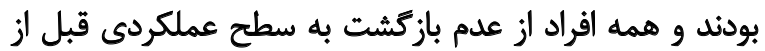

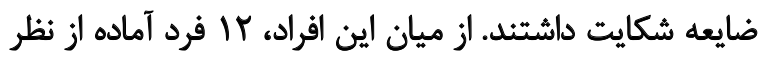

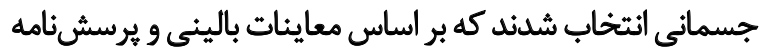

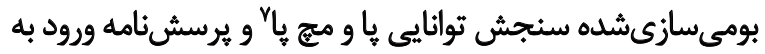

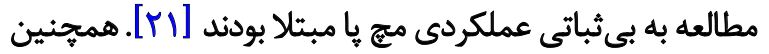

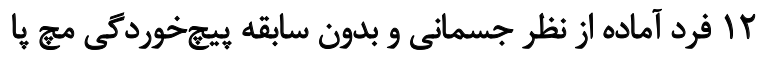

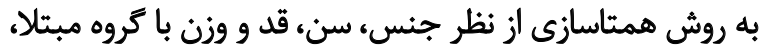

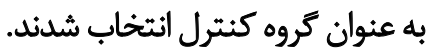

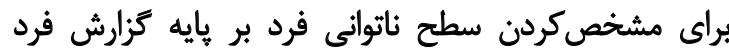

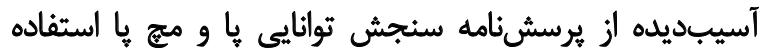

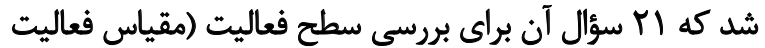

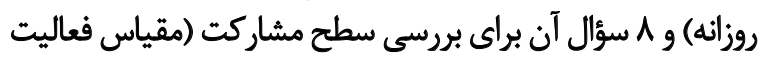

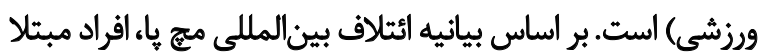

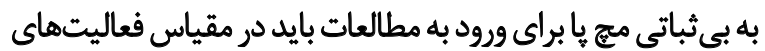

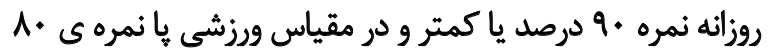

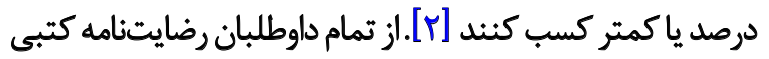

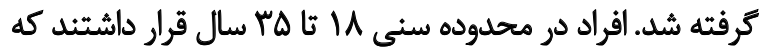
به مدت حداقل ץ روز در هفته فعاليت ورزشى داشتئد نبود بيىثباتى مكانيكى در مفصل مجي ها با استفادهاز آزمايش هائ

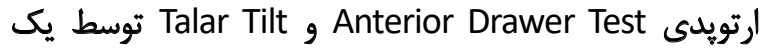
فيزيوتراييست مجرب ارزيابى شد (بر اساس معيار ينج درجهاي

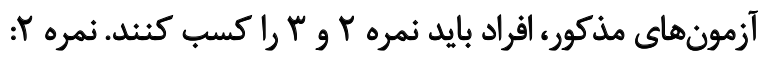

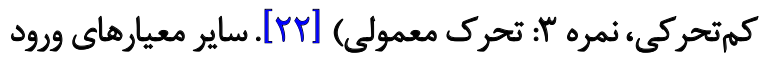

7. Foot and Ankle Ability Measure (FAAM) 
جدول (. اطلاعات جمعيتشئاختى افراد شركت كنثده (ميائكين \pm انحرافمعيار)

\begin{tabular}{|c|c|c|}
\hline (FAI) & كروه كتترل (CON) & مثير \\
\hline 9.8 & $V_{c} \Delta$ & جنس (زن، مرد) \\
\hline$r \Delta / F \mid \pm \Delta / T r$ & $r F / I E \pm T / F$. & سن (سال) \\
\hline$g F / g g \pm I T / N T$ & $g=/ Y \Delta \pm|r / \Lambda|$ & وزن (كيلوكرم) \\
\hline$M M \pm \cdot N r$ & $1 / N \Delta \pm . / .9$ & قد (متر) \\
\hline$\varepsilon \Delta / r V \pm 19 / 19$ & $1 . . \pm \%$ & سنجش توانايى باو مهج يا (مقياس ورزنى) \\
\hline$A+/ 9 Y \pm V / \% \&$ & $1 . * \pm * /$ & سنجش توانايى ها و مج ها (مقياس روزانه) \\
\hline$V \cdot * \pm \Delta / M r$ & $N \cdot N \pm P / N$ & 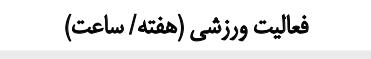 \\
\hline$V / \cap Y \pm r / F A$ & - & خالىشلن همج يا (سال/ تعداد) \\
\hline
\end{tabular}

در هر دو حالت راهرفتن و متغير زمان قدم نسبت به افراد كروه سالم نشان دادند (جدول شماره (T).

Latiol بـ

نتايج مطالعه حاضر نشان داد تحميل تكليف شناختى حين

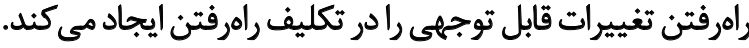

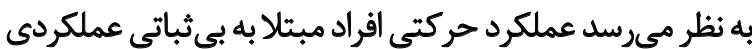

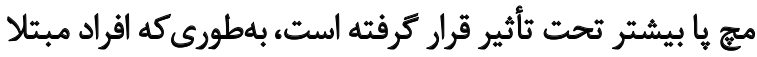

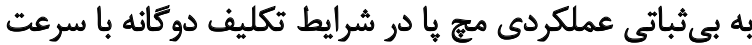

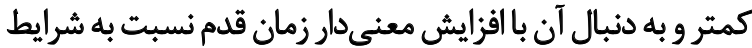

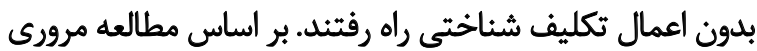

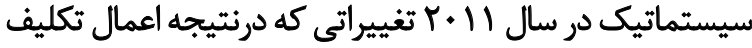

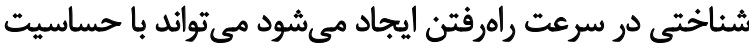

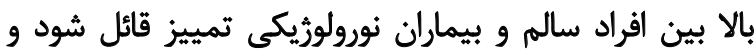

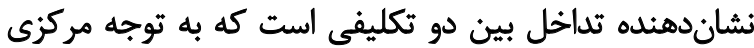

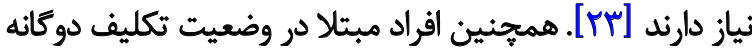

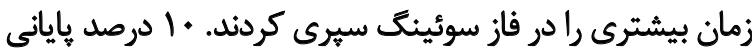

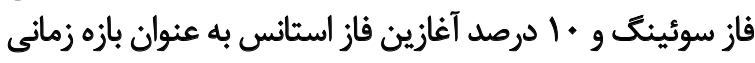

در جدول شماره الطلاعات جمعيتشناختى افرادشركت كنينده

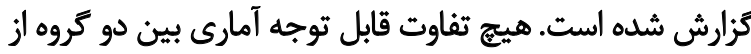

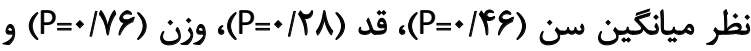

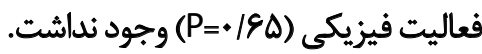

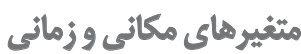
كروه مبتلا به بيىثباتى عملكردى ميج يّا در سرعت قدم كاهش

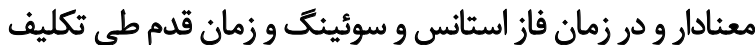

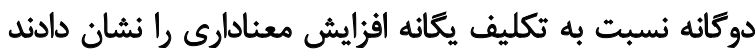

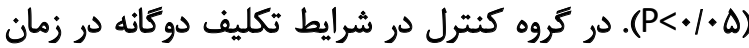

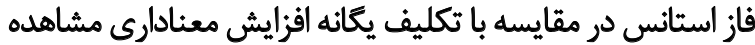

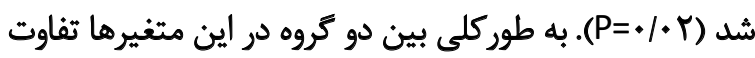

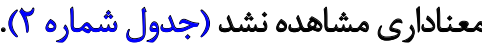
تثييريذيرى اقراد مبتلا به بي ثباتى عملكردى مج يا تغييريذيرى بيشترى را

جدول ץ. مقايسه متغيرهاى راهرفتن در دو حالت تكليف يعانه و دوكانه (انحرافمعيار 士 ميانكين)

\begin{tabular}{|c|c|c|c|c|c|c|c|c|}
\hline \multicolumn{4}{|c|}{ سطح معنادارى (P*) } & \multicolumn{2}{|c|}{ كروه مبتالا (FAI) } & \multicolumn{2}{|c|}{ كروه كتترل (CON) } & \multirow[b]{2}{*}{ متغير } \\
\hline $\begin{array}{l}\text { FST/ } \\
\text { FDT }\end{array}$ & $\begin{array}{l}\text { CST/ } \\
\text { CDT }\end{array}$ & $\begin{array}{l}\text { CDT/ } \\
\text { FDT }\end{array}$ & $\begin{array}{l}\text { CST/ } \\
\text { FST }\end{array}$ & شمراهتى تكليف & شنداختى تكليف (ST) & 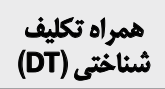 & شُناختي تكليف (ST) & \\
\hline .1 .5 & $.1 \cdot r$ & $\cdot / \pi$ & .18 & $V / / V \pm \Delta / \pi T$ & ES/RTIF/RA & $V \Delta / R+ \pm \mid F / F \Delta$ & $G N \cdot A \pm Q / A V$ & زمان فاز استانس (ms) \\
\hline .1 .1 &.$/ P A$ &.$/ 1 Y$ & r & $r T / A r \pm Y / A Y$ & $P \cdot / T Y \pm Y / M A$ & $P \Delta / \Delta \cdot \pm \Delta / Q 1$ & $P r / A r \pm 8 / p q$ & زمان فاز سوئينك (ms) \\
\hline .1 .4 & $\% r$ &.$/ 4 T$ &.$/ 1 \Delta$ & $\Delta V / Y \pm \pm / A q$ & $\Delta T / Q Y \pm T / \Delta$. & $8 \cdot / \pi+1 \cdot 1 \cdot 1$ & $\Delta Q / q Y \pm E / F i$ & زمان قدم (ms) \\
\hline.$/ 91$ & $\cdot \mathrm{MA}$ & .198 & $.19 V$ & $g N \cdot r \pm q q / A \mid$ & $8 / g / 4 \cdot \pm n / \cdot \Delta$ & STNQYITNHI & 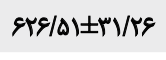 & طول قدم (mm) \\
\hline $.1 \% 5$ & . & $\cdot / A F$ &.$/ \Delta \Delta$ & $V / * A \pm \cdot / Y$ & $1 / / \vee \pm \cdot / 1 \varepsilon$ & $V / v \pm * / / V$ & $1 / / r \pm \cdot / T$ & سرعت (m/s) \\
\hline- & - & - & - & . NE1 &.$/ 48$ &.$/ 9 \mathrm{VA}$ &.$/ 9 \mathrm{r}$ & تغييريذيزي زمان قدم (ICC) \\
\hline
\end{tabular}

ST: Single Task; DT: Dual Task; CST(DT): Control Single (Dual) Task; FST(DT): FAI Single (Dual) Task; (P<•/•ه) 
با جالش تكليف دوكاثه روبهرو شدند استراترى متفلوتى را ارز نظر تغييريذيرى زمان قدم نشان دادند.

كروه مبتلا با اعمال تكليف شناختى كاهش تغييريذيرى

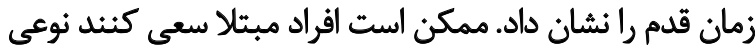

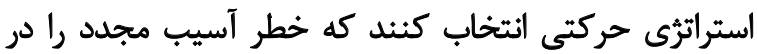

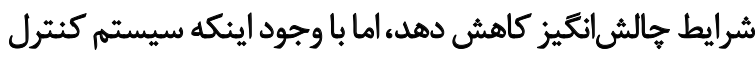

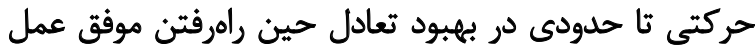

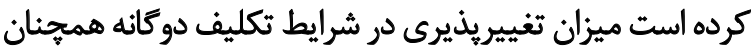

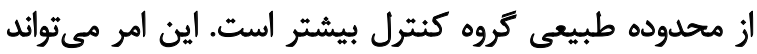

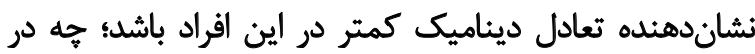

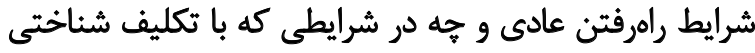

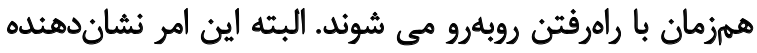

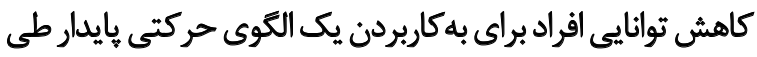

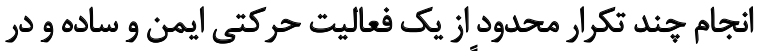

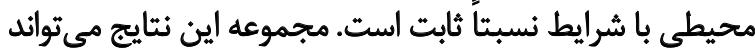

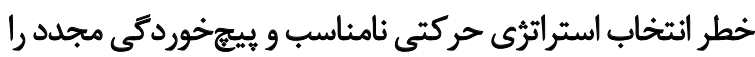
در افراد مبتلا به بي ثباتى عملكردى مج يا يا افزايش دهدي.

متأسفانه مطالعات اندكى اثر اعمال تكليف شناختى حين

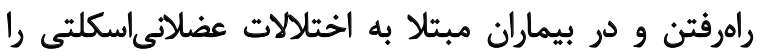

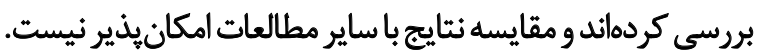

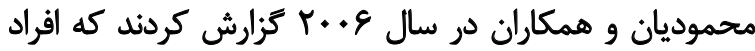

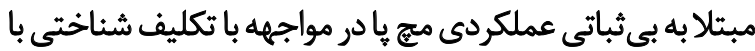

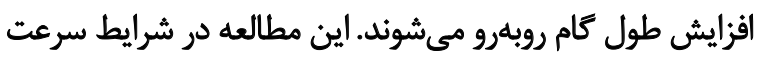

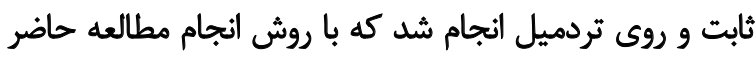

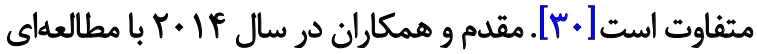

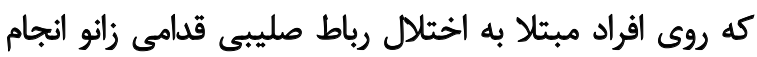

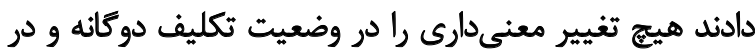

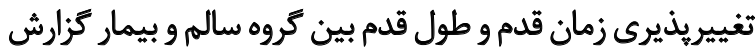

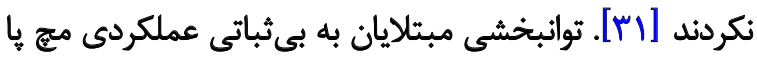

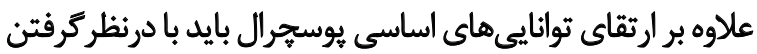

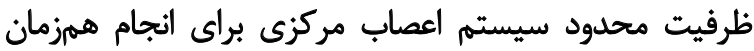

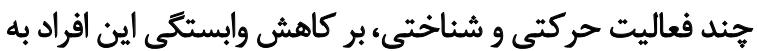
يردازش آكاهانه اطلاعات نيز تمركز كند.

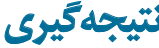

با توجه به نتايج بهدستآمده از بررسى حاضر مشخص شئ افر افراد

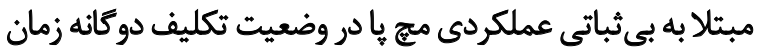

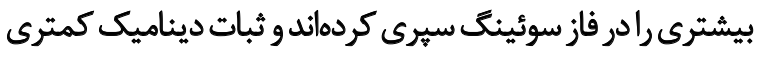

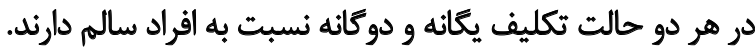

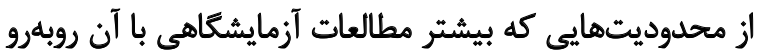

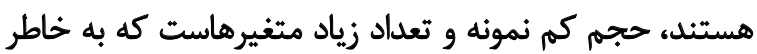

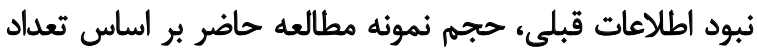

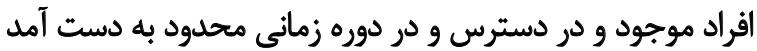

مهم در افراد مبتلا به بى ثباتى مج يا مطرح است؛ جرا كه بيشتر

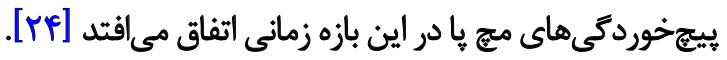
مطالعات نشان مى دهد انتخاب اطلاعات حسى مختلف توسط

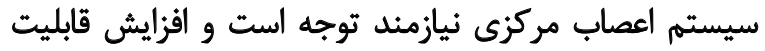

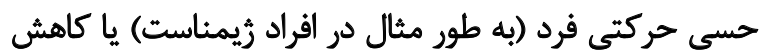

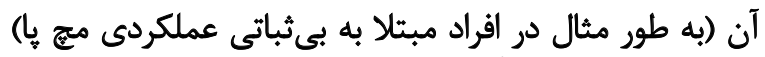

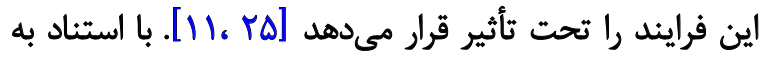

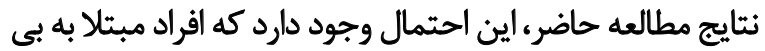

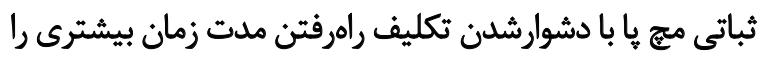

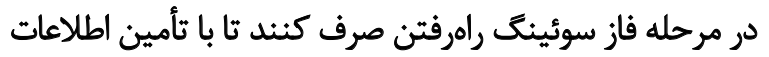

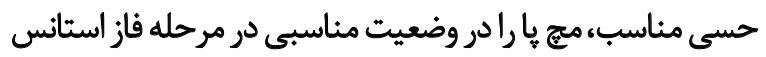

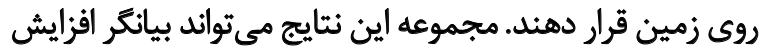
نيازهاى توجهى راهرفتن در افراد مبتلا باشد.

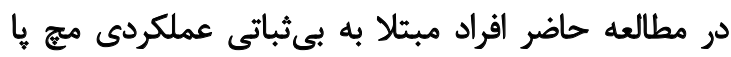
تغييريذيرى بيشترى را هنكام راهرفتن با سرعت عادى نسبت بـاني

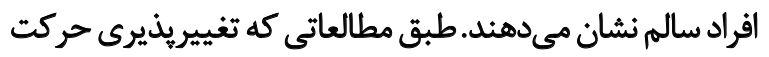

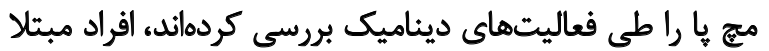

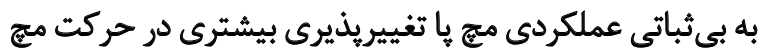

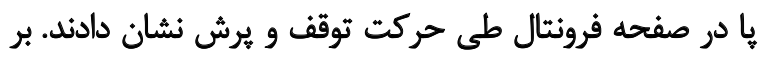

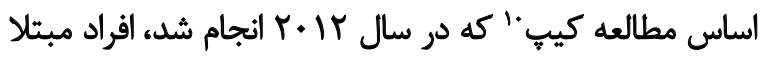

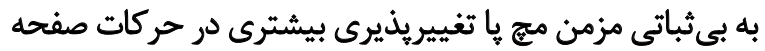

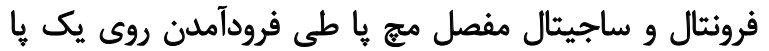

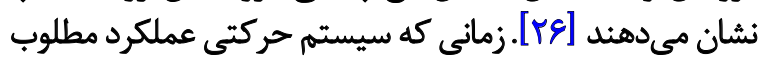

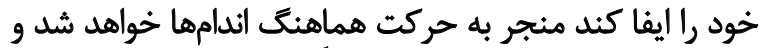

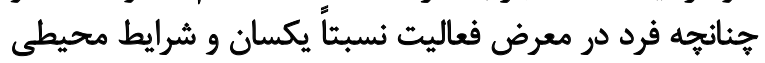

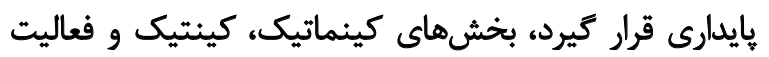

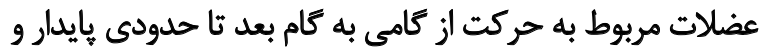

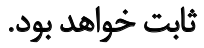

در سيستمهاى بيولوريكى تغييريذيرى كمتر از حد مطلوب

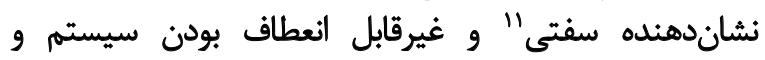

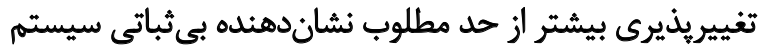

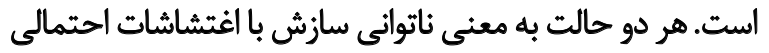

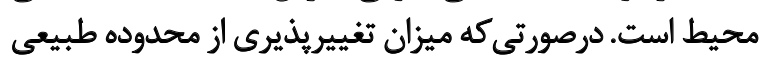

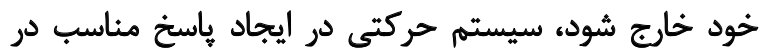

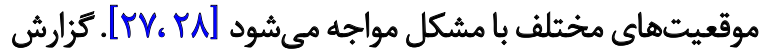

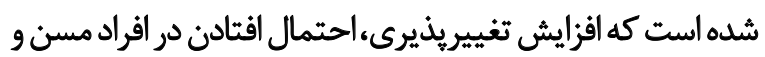

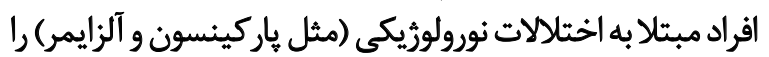

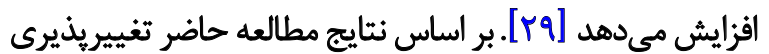

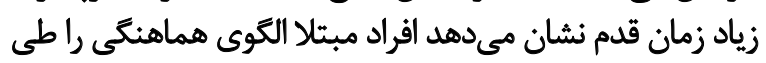

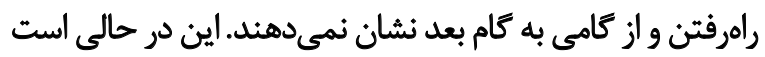

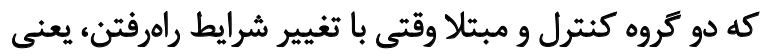

\section{Kipp}

11. Stiffness 


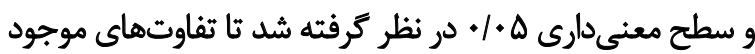

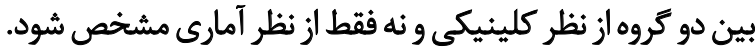

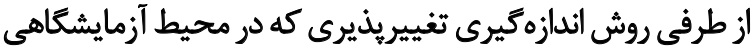

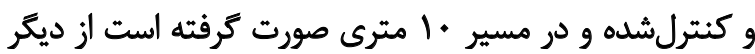

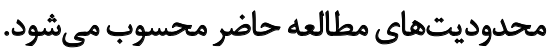

در مطالعه حاضر از فعاليت راهرفتن استفاده شد كه در مقايسه

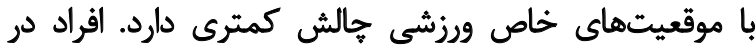

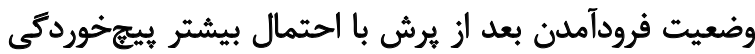

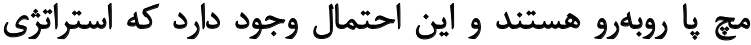

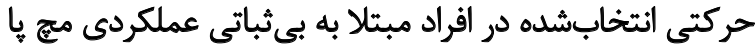

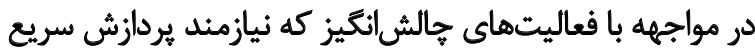

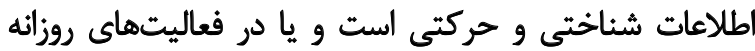

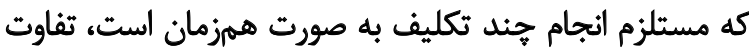

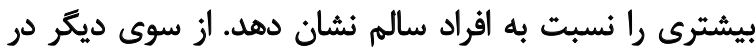

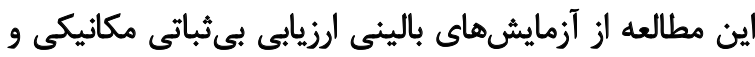

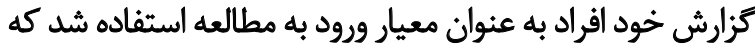

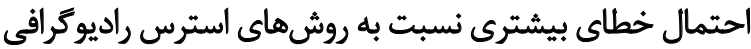

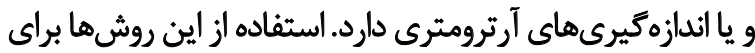

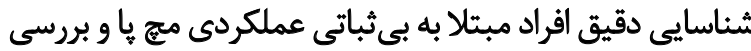

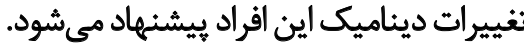

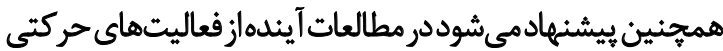

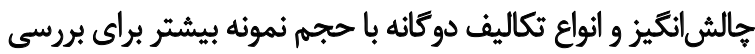

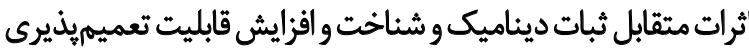

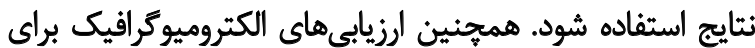

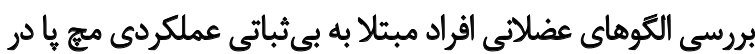

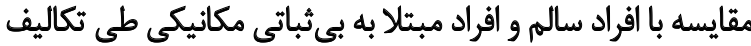

دوكانه و جندكانه ضرورى به نظر مىرسد.

$$
\text { تشكر وقدرداني }
$$

اين بروره با بودجه تحقيقاتى داتشكاه علوم يزشكى اصفهان

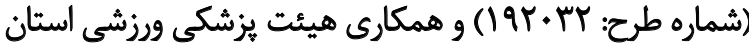

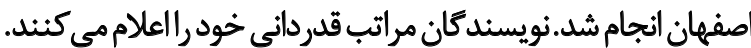




\section{References}

[1] Anandacoomarasamy A. Long term outcomes of inversion ankle injuries: Commentary. British Journal of Sports Medicine. BMJ; 2005; 39(3):14. doi: 10.1136/bjsm.2004.011676

[2] Gribble PA, Delahunt E, Bleakley C, Caulfield B, Docherty C, Fourchet F, et al. Selection criteria for patients with chronic ankle instability in controlled research: A position statement of the International Ankle Consortium: Table 1. British Journal of Sports Medicine. BMJ; 2013; 48(13):1014-8. doi: 10.1136/bjsports-2013-093175

[3] Munn J, Sullivan SJ, Schneiders AG. Evidence of sensorimotor deficits in functional ankle instability: A systematic review with meta-analysis. Journal of Science and Medicine in Sport. 2010; 13(1):2-12. doi: 10.1016/j.jsams.2009.03.004

[4] Kazemi K, Abdollahi I, Arab A. Evaluation of the electromyographic activity of distal and proximal muscles of the lower extremity after ankle sprain (Review article). Physical Treatments. 2013; 3(3):46-52

[5] Gutierrez GM, Knight CA, Swanik CB, Royer T, Manal K, Caulfield $\mathrm{B}$, et al. Examining neuromuscular control during landings on a supinating platform in persons with and without ankle instability. The American Journal of Sports Medicine. 2011; 40(1):193201. doi: $10.1177 / 0363546511422323$

[6] Hertel J. Sensorimotor deficits with ankle sprains and chronic ankle instability. Clinics in Sports Medicine. 2008; 27(3):353-70. doi: 10.1016/j.csm.2008.03.006

[7] Brown C. Foot Clearance in walking and running in individuals with ankle instability. American Journal of Sports Medicine. 2011; 39(8):1769-77. doi: 10.1177/0363546511408872

[8] Delahunt E, Monaghan K, Caulfield B. Altered neuromuscular control and ankle joint kinematics during walking in subjects with functional instability of the ankle joint. The American Journal of Sports Medicine. 2006; 34(12):1970-6. doi: 10.1177/0363546506290989

[9] Herb CC, Chinn L, Dicharry J, McKeon PO, Hart JM, Hertel J. Shank-rearfoot joint coupling with chronic ankle instability. Journal of Applied Biomechanics. Human Kinetics; 2014; 30(3):36672. doi: 10.1123 /jab.2013-0085

[10] Abbud GAC, Li KZH, DeMont RG. Attentional requirements of walking according to the gait phase and onset of auditory stimuli. Gait \& Posture. 2009; 30(2):227-32. doi: 10.1016/j.gaitpost.2009.05.013

[11] Huxhold O, Li SC, Schmiedek F, Lindenberger U. Dual-tasking postural control: Aging and the effects of cognitive demand in conjunction with focus of attention. Brain Research Bulletin. 2006; 69(3):294-305. doi: 10.1016/j.brainresbull.2006.01.002

[12] Hayati M, Ashayeri H, Salavati M, Saraf-Zadeh J, Keyhani M R. [Comparison of postural stubility and auditory short- term memory task interference between patients with functional ankle instability and healthy individuals (Persian)]. Archives of Rehabilitation. 2010; 11(1):34-9

[13] Soltani M, Negahban H, Mehravar M, Tajali S, Hessam M. The effect of dual tasking on the lower extremity kinematics during lo- comotion in patients with anterior cruciate ligament injury. Physical Treatments. 2013; 3(1):56-66.

[14] Rahnama L, Akhbari B, Salavati M, Kazem-Nezhad A. [Comparison of attentional demands of postural control between ath letes wieh functional ankle instability and healthy athletes (Persian)]. Archives of Rehabilitation. 2008; 9(3-4):38-42.

[15] Shiravi Z, Talebian S, Hadian M, Oliaie G. Effect of cognitive task on postural control of the patients with chronic ankle sprain. Journal of Foot and Ankle Research. 2012; 5(Suppl 1):24. doi: 10.1186/1757-1146-5-s1-p24

[16] Burcal CJ, Wikstrom EA. Cognitive loading-induced sway alterations are similar in those with chronic ankle instability and uninjured controls. Gait \& Posture. 2016; 48:95-8. doi: 10.1016/j. gaitpost.2016.05.004

[17] Terada M, Bowker S, Thomas AC, Pietrosimone B, Hiller CE, Rice MS, et al. Alterations in stride-to-stride variability during walking in individuals with chronic ankle instability. $\mathrm{Hu}$ man Movement Science. 2015; 40:154-62. doi: 10.1016/j.humov.2014.12.004

[18] Springer S, Giladi N, Peretz C, Yogev G, Simon ES, Hausdorff JM. Dual-tasking effects on gait variability: The role of aging, falls, and executive function. Movement Disorders. 2006; 21(7):950-7. doi: $10.1002 / \mathrm{mds} .20848$

[19] Menant J, Steele J, Menz H, Munro B, Lord S. Step time variability and pelvis acceleration patterns of younger and older adults: effects of footwear and surface conditions. Research in Sports Medicine. 2011; 19(1):28-41. doi: 10.1080/15438627.2011.534968

[20] Hausdorff JM. Gait dynamics, fractals and falls: Finding meaning in the stride-to-stride fluctuations of human walking. $\mathrm{Hu}$ man Movement Science. 2007; 26(4):555-89. doi: 10.1016/j.humov.2007.05.003

[21] Mazaheri M, Salavati M, Negahban H, Sohani SM, Taghizadeh F, Feizi A, et al. Reliability and validity of the Persian version of Foot and Ankle Ability Measure (FAAM) to measure functional limitations in patients with foot and ankle disorders. Osteoarthritis and Cartilage. 2010; 18(6):755-9. doi: 10.1016/j.joca.2010.03.006

[22] Ryan L. Mechanical stability, muscle strength and proprioception in the functionally unstable ankle. Australian Journal of Physiotherapy. 1994; 40(1):41-7. doi: 10.1016/s0004-9514(14)60453-0

[23] Al-Yahya E, Dawes H, Smith L, Dennis A, Howells K, Cockburn J. Cognitive motor interference while walking: A systematic review and meta-analysis. Neuroscience \& Biobehavioral Reviews. 2011; 35(3):715-28. doi: 10.1016/j.neubiorev.2010.08.008

[24] Drewes LK, McKeon PO, Paolini G, Riley P, Kerrigan DC, Ingersoll CD, et al. Altered ankle kinematics and shank-rearfoot coupling in those with chronic ankle instability. Journal of Sport Rehabilitation. Human Kinetics; 2009; 18(3):375-88. doi: 10.1123/jsr.18.3.375

[25] Vuillerme N, Nougier V. Attentional demand for regulating postural sway: The effect of expertise in gymnastics. Brain Research Bulletin. 2004; 63(2):161-5. doi: 10.1016/j.brainresbull.2004.02.006

[26] Kipp K, Palmieri-Smith RM. Principal component based analysis of biomechanical inter-trial variability in individuals with 
chronic ankle instability. Clinical Biomechanics. 2012; 27(7):70610. doi: 10.1016/j.clinbiomech.2012.02.005

[27] Davids K, Glazier P, Arajo D, Bartlett R. Movement systems as dynamical systems. Sports Medicine. 2003; 33(4):245-60. doi: 10.2165/00007256-200333040-00001

[28] Stergiou N, Harbourne RT, Cavanaugh JT. Optimal movement variability. Journal of Neurologic Physical Therapy. 2006; 30(3):120-9. doi: 10.1097/01.npt.0000281949.48193.d9

[29] Hausdorff JM. Gait variability: Methods, modeling and meaning. Journal of NeuroEngineering and Rehabilitation. 2005; 2(1):19. doi: 10.1186/1743-0003-2-19

[30] Mahmoudian A, Akhbari B, Salavati M. [Attentional demands of walking in athletes with and without functional ankle instability (Persian)]. Archives of Rehabilitation. 2010; 10(4):40-5.

[31] Nazary-Moghadam S, Akhbari B, Esteki A, Salavati M, Keyhani S, Zeinalzadeh A. Effect of dual-tasking on variability of spatiotemporal parameters in subjects with and without anterior cruciate ligament deficiency using linear dynamics. Physical Treatments. 2015; 4(4):213-220. 
